\title{
Prevalence of and risk factors for depression among female Syrian refugees and Jordanians with chronic disease: a pilot study
}

\author{
Madison Sharp, ${ }^{1}$ Alyssa Parpia, ${ }^{2}$ Mamoun Ahram, ${ }^{3}$ Refqi Mahmoud ${ }^{4}$ and Kaveh Khoshnood ${ }^{2}$
}

${ }^{1}$ Yale School of Medicine; ${ }^{2}$ Department of Epidemiology of Microbial Diseases, Yale School of Public Health, New Haven, United States of America (Correspondence to: Kaveh Khoshnood: kaveh.khoshnood@yale.edu). ${ }^{3}$ Department of Physiology and Biochemistry, School of Medicine, University of Jordan, Amman, Jordan. ${ }^{4}$ Division of Cardiovascular Disease, Jordanian Ministry of Health, Amman, Jordan

\begin{abstract}
Background: Chronic disease and depression are primary contributors to morbidity among displaced and local populations.

Aims: This study aims to address the prevalence for and risk factors of comorbid depression among Jordanian and Syrian women with known chronic disease.

Methods: To provide evidence for mental health intervention planning, we conducted a cross-sectional survey to investigate determinants of depression among female Syrian refugees and Jordanians with chronic disease living in Amman. A total of 272 female Syrian refugees and Jordanians with chronic disease were recruited from 4 clinics across Amman from June to August 2017. We compared demographic and health characteristics and depression level and identified predictors of depression via multivariable ordinal regression.

Results: Moderate to high levels of depression were reported in $55.9 \%$ of the participants, with a prevalence of $41.1 \%$ among Jordanians and $70.6 \%$ among Syrians. Syrians with chronic disease had 2.73 times greater odds of higher levels of depression than their Jordanian peers. After adjusting for age, income, spouse employment status, gastrointestinal or genitourinary disorder status, and perceived self-efficacy, Syrians were not at significantly greater odds of reporting higher levels of depression. Risk factors for higher depression levels included having an unemployed spouse, diagnosis of gastrointestinal or genitourinary disorder and low perceived self-efficacy.

Conclusion: Depression is frequently comorbid with chronic physical conditions and has a deleterious impact on health status. Mental health interventions and chronic disease management tailored to differences among local and displaced communities may reduce disease burden and disability.

Key words: depression, prevalence, risk factors, female refugees, Syrian, Jordan

Citation: Sharp M; Parpia A; Ahram M; Mahmoud R; Khoshnood K. Prevalence of and risk factors for depression among female Syrian refugees and Jordanians with chronic disease: a pilot study. East Mediterr Health J. 2021;27(12):1142-1152. https://doi.org/10.26719/emhj.20.123

Received: 04/10/19; accepted: 12/05/20

Copyright (C) World Health Organization (WHO) 2021 Open Access. Some rights reserved. This work is available under the CC BY-NC-SA 3.0 IGO license (https://creativecommons.org/licenses/by-nc-sa/3.o/igo).
\end{abstract}

\section{Introduction}

\section{Background}

The ongoing Syrian civil war, which began in 2011, constitutes the largest refugee and displacement crisis since World War II. More than 5.6 million Syrians have fled to surrounding countries, settling primarily in Turkey, Lebanon and Jordan (1). As of January 2019, the Office of the United Nations High Commissioner for Refugees in Jordan has registered over 670000 Syrian refugees (1). The majority of Syrian refugees in Jordan live in urban areas, with women aged 18-59 years comprising nearly a quarter of this population (1).

The protracted nature of the Syrian civil war has forced Jordanian health professionals to contend with the complexity of managing chronic conditions with limited resources. The rising prevalence of noncommunicable diseases worldwide increases the likelihood that Syrian refugees arriving in Jordan suffer from pre-existing medical conditions (2). In addition to increased chronic illnesses, high rates of mental health disorders secondary to trauma and violence place an enormous burden on the Jordanian health care system. Female refugees with chronic disease may be at especially high risk of mental illness (3), yet the prevalence and predictors of depression for this vulnerable population have not been well researched. Moreover, studies on the mental health of Middle Eastern women rarely acknowledge the heterogeneity of the Middle East (4), and research stratified by nationality is critical in order to elucidate differences across this diverse region.

Jordanians have also experienced the epidemiological transition from primarily communicable diseases to noncommunicable diseases over the past few decades (5). As a result, local populations remain at substantial risk of developing chronic diseases and mental health disorders. In fact, a $20 \%$ prevalence of depression among Jordanians with chronic disease (6) and a 35\% prevalence of depression among camp-based Syrian refugees with chronic disease in Jordan (7) have been reported previously. However, studies examining chronic disease and depression among non-camp refugees are lacking. 
Given that more than $80 \%$ of Syrian refugees in Jordan live outside refugee camps (1), with nearly a third residing in the Amman Governorate, comparing risk factors for depression among non-camp Syrian refugees in Amman and Jordanians may help inform future studies and interventions targeted at individual groups.

The bidirectional relationship between depression and chronic disease has been well documented (8). Comorbid depression has a deleterious impact on the health of chronic disease patients (8). Additionally, patients with chronic disease often report higher rates of depression. In light of the increasing burden of noncommunicable diseases and depression faced by displaced populations, particularly women, we undertook this study to characterize the prevalence of, and predictors for, depression among female Jordanians and Syrian refugees in non-camp settings.

\section{Objectives}

Among female Jordanian and Syrian refugees with known chronic disease, we sought to define the prevalence of depression, the predictors of depression, and the association between chronic disease and depression, with the goal of determining the feasibility of a larger scale study. Because Syrian refugees and Jordanians receive medical aid through different and evolving health care systems, this study also seeks to identify differences in the predictors of comorbid physical and mental noncommunicable diseases in order to assist service providers and policy-makers.

\section{Methods}

\section{Data collection}

A total of 272 female Jordanians and Syrian refugees recruited from 4 clinics and hospitals in Amman, Jordan participated in the study. Jordanians were recruited primarily from Jordan University Hospital and Amman Medical Center. Syrians were mainly recruited from Caritas and the Institute of Family Health clinic in East Amman. Data were collected from June until August 2017 using the Patient Health Questionnaire-Somatic, Anxiety, and Depressive Symptoms (PHQ-SADS), Multidimensional Scale of Perceived Social Support (MSPSS), General Self-Efficacy Scale (GSE), along with demographic and chronic disease surveys. All questionnaires were translated into Arabic and back-translated into English.

These clinical settings were selected because they represent sites of care for underserved Jordanians and Syrians in Amman. The University of Jordan Hospital is a large academic hospital and Amman Medical Center is a Ministry of Health clinic. Concerning Syrian refugee health care, the Jordanian government significantly increased the fees at Ministry of Health facilities in 2014 after previously providing subsidized health insurance to these refugees $(13,14)$. As a result, many refugees now seek care at nongovernmental clinics such as Caritas and the Institute of Family Health clinic.
To ensure homogenous data collection and reduce errors, a female Jordanian interpreter assisted with survey completion. After obtaining written consent, Arabic questionnaires were distributed to the participants to self-complete. Data collection occurred in waiting rooms in hospitals or clinics, and the questionnaires took about 20 minutes to complete. Illiterate participants were guided by the interpreter, who recorded their responses.

Participants were recruited for this cross-sectional study using convenience sampling. The inclusion criteria for the study were: female Jordanian or Syrian refugee who arrived in Jordan since the start of the Syrian conflict, received medical care at a clinic or hospital in Amman, had at least one chronic disease, and aged 18-50 years. Pregnant women were excluded. Ethical approval to conduct the study was obtained from the Yale Human Subjects Committee, Jordanian Ministry of Health, Caritas Jordan, Noor Al-Hussein Foundation and Jordan University Hospital.

\section{Dependent and independent variables}

The Patient Health Questionnaire-9 (PHQ-9), which was employed as this study's main outcome measure, is a multipurpose instrument for screening and diagnosing severity of depression. The tool, consisting of the $9 \mathrm{Di}$ agnostic and Statistical Manual of Mental Disorders, 4th ed (DSM-IV) criteria for major depressive disorder, contains 8 questions to assess depressive symptoms and one question to assess functional impairment. Each of the 9 items is scored from o (not at all) to 3 (nearly every day) (15). None/low, moderate, and high depressive symptoms correspond to scores of $0-9,10-18$, and 19-27, respectively. These levels are consistent with a meta-analysis that found a cut-off score between 8 and 11 was acceptable for diagnosing depression (16).

Nationality, the primary independent variable in this analysis, serves as a proxy for forced displacement. Additional demographic variables analysed included age, education level, number of family members, occupation, income, education, husband's occupation and housing status. All variables were converted to well-distributed categorical variables. Binary variables were created for all chronic disease data, which included 6 variables for diagnosis of each type of chronic disease, treatment status and length of treatment. The 6 categories of chronic diseases included: cardiometabolic, respiratory, neurologic/cancer, rheumatology, endocrine and gastrointestinal/genitourinary (GI/GU).

A series of questionnaires was used to identify level of social support, perceived self-efficacy, somatic symptom severity, anxiety and depression in our study population. The MSPSS is a 12-item tool to evaluate perceived adequacy of social support, an important counter to stressful life events (17). The GSE is a 10-item scale used to assess perceived self-efficacy (18). Both the MSPSS and GSE have been validated among Arab women $(17,18)$. The PHQ-SADS comprises 3 validated questionnaires: the Patient Health Questionnaire-15 (PHQ-15), the Generalized Anxiety Disorder-7 (GAD-7), and the 
PHQ-9. The internal consistency reliability measured using Cronbach's alpha for the Arabic versions of the PHQ-15, GAD-7 and PHQ-9 was 0.826, 0.763 and 0.857 , respectively (19). The PHQ-15 is used to identify somatization, a substantial contributor to functional impairment. The GAD-7 is a 7-item screening tool for anxiety $(19,20)$.

\section{Statistical analysis}

Previous studies have found a $20 \%$ prevalence of depression among Jordanians with chronic disease (6) and a $35 \%$ prevalence of depression among Syrian refugees in Jordan with chronic disease (7). In order to achieve $80 \%$ power to detect a $15 \%$ difference in prevalence of depression between Jordanians and Syrian refugees, this study required 136 Jordanians and 136 Syrians, a total of 272 participants.

Descriptive statistics on all variables were computed for the total study population, as well as separately for each nationality for the populations of Jordanians and Syrians. Bivariate analysis by nationality was performed for each variable against level of depression, our main outcome variable. Level of depression is an ordinal categorical variable having 3 levels: none/low, moderate and high. Hypothesis testing was conducted using chi-squared tests with continuity correction for categorical variables, and analysis of variance (ANOVA) was performed for continuous variables with an equal variance assumption. For categories with less than 5 individuals, Fisher's exact test was used for categorical variables and the KruskalWallis test for continuous variables.

We performed multivariable ordinal logistic regression for the outcome variable of level of depression, with the assumption that the difference between none/ low and moderate depression is equal to the difference between moderate and high depression. Predictor variables were selected on the basis of their bivariate association with the outcome variable in the 2 study populations of Jordanians and Syrians living in Jordan, as well as the variables that were found to be significantly associated in the literature $(6,21-23)$. For each predictor variable identified in the multiple ordinal regression model, adjusted odds ratios (OR) and their associated $95 \%$ confidence intervals (CI) were obtained. Data were analysed using R-3.4.4.

\section{Results}

\section{Population characteristics of female Jordanians and Syrians stratified by nationality}

Participants were typically between $35-50$ years old, married, educated, and unemployed (Table 1). The majority of patients had no more than one chronic disease (57.0\%), the most common of which were cardiometabolic (41.9\%), rheumatologic $(38.2 \%)$ and endocrine $(23.2 \%)$ conditions. Most patients reported either none/low (44.1\%) or moderate $(44.1 \%)$ depression, moderate somatic symptoms
(60.7\%), and moderate anxiety (42.6\%) as determined through validated survey instruments. Overall, the cohort had moderate/high perceived self-efficacy (64.0\%) and high perceived social support (80.9\%) (Table 1).

The Jordanian women had higher levels of education (more than primary school) (95.6\% vs $47.8 \%$; $P<0.001$ ), home ownership (59.6\% vs $0.0 \% ; P<0.001)$, employment (28.7 vs. $0.7 \% ; P<0.001$ ), and income (95.6\% v. $50.7 \% . P$ $<0.001$ ) than the Syrians (Table 1). We found a lower proportion of Jordanians were married compared with Syrians (73.5\% vs 92.6\%; $P<0.001)$, yet a greater proportion of Jordanians had a spouse who was employed (65.4\% vs 41.2\%; $P<0.001$ ).

Syrians had a higher overall chronic disease burden (52.9\% vs 33.1\%; $P=0.001$ ), categorized by the number of chronic disease diagnoses, and the higher incidence of cardiometabolic disease $(50.7 \%$ vs $33.1 \% ; P=0.005)$, rheumatologic disease $(47.1 \%$ vs $29.4 \% ; P=0.004)$ and GI/GU disorders (25.7\% vs $11.0 \%$; $P=0.003$ ) than Jordanians (Table 1). However, Syrians reported lower rates of endocrine disease $(15.4 \%$ vs $30.9 \% ; P=0.005$ ) and neurologic disease/cancer (5.1\% vs $13.2 \% ; P=0.036$ ) compared with Jordanians. Syrians more frequently reported experiencing moderate to high levels of depression $(P<0.001)$, somatic symptoms $(P<0.001)$ and anxiety $(P<0.001)$ than Jordanians. Syrians also reported lower levels of perceived self-efficacy $(50.0 \%$ vs $22.1 \%$; $P$ $<0.001)$ and social support ( $24.3 \%$ vs $14.0 \% ; P=0.045)$.

\section{Depression prevalence among female Jordanians and Syrians}

Overall, 120 (44.1\%) participants reported none/low levels of depression, $120(44.1 \%)$ reported moderate levels of depression, and 32 (11.8\%) reported high levels of depression (Table 1). Our results show that 41 (30.1\%) Jordanians had moderate depression and $15(11.0 \%)$ had severe depression compared to $79(58.1 \%)$ and 17 (12.5\%) Syrians, respectively. Eighty (58.8\%) Jordanians and 40 (29.4\%) Syrians reported none/low depression levels, respectively.

\section{Population characteristics of female Jordanians and Syrian refugees stratified by nationality and depression level}

We found no significant association between level of depression and participants' age, marital status, number of family members, employment status, spouse's occupation, chronic disease burden, chronic disease classification (except for GI/GU), receiving treatment and duration of treatment (Table 2). However, higher PHQ-15 severity, higher GAD-7 severity, and lower MSPSS scores were significantly associated with level of depression and nationality among both cohorts. Among Jordanians, our analysis identified significant associations between depression level and lower income level $(P=0.014)$, renting instead of owning a house $(P=0.033)$ and lower GSE score $(P=$ 0.041). Statistically significant differences in level of depression were seen for lack of spouse employment $(P=$ 0.006), spouse occupation $(P=0.017)$ and diagnosis of $\mathrm{GI} /$ GU disease $(P=0.003)$ among Syrians (Table 2$)$. 


\begin{tabular}{|c|c|c|c|c|c|c|c|}
\hline \multirow[t]{2}{*}{ Characteristic } & \multicolumn{2}{|c|}{ Total $(n=272)$} & \multicolumn{2}{|c|}{ Jordanian $(n=136)$} & \multicolumn{2}{|c|}{ Syrian $(n=136)$} & \multirow[t]{2}{*}{$P$-value } \\
\hline & No. & $\%$ & No. & $\%$ & No. & $\%$ & \\
\hline Age (years) & & & & & & & 0.574 \\
\hline $18-34$ & 67 & 24.6 & 36 & 26.5 & 31 & 22.8 & \\
\hline $35-50$ & 205 & 75.4 & 100 & 73.5 & 105 & 77.2 & \\
\hline Marital status & & & & & & & $<0.001$ \\
\hline Not married & 46 & 16.9 & 36 & 26.5 & 10 & 7.4 & \\
\hline Married & 226 & 83.1 & 100 & 73.5 & 126 & 92.6 & \\
\hline No. of family members & & & & & & & 0.057 \\
\hline $1-6$ & 197 & 72.4 & 106 & 77.9 & 91 & 66.9 & \\
\hline $7-12$ & 75 & 27.6 & 30 & 22.1 & 45 & 33.1 & \\
\hline Education level & & & & & & & $<0.001$ \\
\hline No education/primary & 77 & 28.3 & 6 & 4.4 & 71 & 52.2 & \\
\hline More than primary & 195 & 71.7 & 130 & 95.6 & 65 & 47.8 & \\
\hline Employed & & & & & & & $<0.001$ \\
\hline Yes & 40 & 14.7 & 39 & 28.7 & 1 & 0.7 & \\
\hline No & 232 & 85.3 & 97 & 71.3 & 135 & 99.3 & \\
\hline Monthly income (JD) & & & & & & & $<0.001$ \\
\hline$<200$ & 73 & 26.8 & 6 & 4.4 & 67 & 49.3 & \\
\hline$\geq 200$ & 199 & 73.2 & 130 & 95.6 & 69 & 50.7 & \\
\hline Housing status & & & & & & & $<0.001$ \\
\hline Own & 81 & 29.8 & 81 & 59.6 & 0 & 0.0 & \\
\hline Rent & 185 & 68.0 & 49 & 36.0 & 136 & 100.0 & \\
\hline Other & 6 & 2.2 & 6 & 4.4 & 0 & 0.0 & \\
\hline Spouse employed & & & & & & & $<0.001$ \\
\hline Yes & 145 & 53.3 & 89 & 65.4 & 56 & 41.2 & \\
\hline No & 79 & 29.0 & 11 & 8.1 & 68 & 50.0 & \\
\hline Not applicable & 48 & 17.6 & 36 & 26.5 & 12 & 8.8 & \\
\hline Spouse occupation & & & & & & & $<0.001$ \\
\hline Government & 45 & 16.5 & 45 & 33.1 & 0 & 0.0 & \\
\hline Private/agricultural & 101 & 37.1 & 45 & 33.1 & 56 & 41.2 & \\
\hline Not applicable & 126 & 46.3 & 46 & 33.8 & 80 & 58.8 & \\
\hline Disease burden & & & & & & & 0.001 \\
\hline 1 disease & 155 & 57.0 & 91 & 66.9 & 64 & 47.1 & \\
\hline$>1$ disease & 117 & 43.0 & 45 & 33.1 & 72 & 52.9 & \\
\hline Cardiometabolic disease & & & & & & & 0.005 \\
\hline No & 158 & 58.1 & 91 & 66.9 & 67 & 49.3 & \\
\hline Yes & 114 & 41.9 & 45 & 33.1 & 69 & 50.7 & \\
\hline Respiratory disease & & & & & & & 0.445 \\
\hline No & 241 & 88.6 & 123 & 90.4 & 118 & 86.8 & \\
\hline Yes & 31 & 11.4 & 13 & 9.6 & 18 & 13.2 & \\
\hline Neurological disease/cancer & & & & & & & 0.036 \\
\hline No & 247 & 90.8 & 118 & 86.8 & 129 & 94.9 & \\
\hline Yes & 25 & 9.2 & 18 & 13.2 & 7 & 5.1 & \\
\hline Rheumatologic disease & & & & & & & 0.004 \\
\hline No & 168 & 61.8 & 96 & 70.6 & 72 & 52.9 & \\
\hline Yes & 104 & 38.2 & 40 & 29.4 & 64 & 47.1 & \\
\hline
\end{tabular}




\begin{tabular}{|c|c|c|c|c|c|c|c|}
\hline \multirow[t]{2}{*}{ Characteristic } & \multicolumn{2}{|c|}{ Total $(n=272)$} & \multicolumn{2}{|c|}{ Jordanian $(n=136)$} & \multicolumn{2}{|c|}{ Syrian $(n=136)$} & \multirow[t]{2}{*}{ P-value } \\
\hline & No. & $\%$ & No. & $\%$ & No. & $\%$ & \\
\hline Endocrine disease & & & & & & & 0.005 \\
\hline No & 209 & 76.8 & 94 & 69.1 & 115 & 84.6 & \\
\hline Yes & 63 & 23.2 & 42 & 30.9 & 21 & 15.4 & \\
\hline GI/GU disease & & & & & & & 0.003 \\
\hline No & 222 & 81.6 & 121 & 89.0 & 101 & 74.3 & \\
\hline Yes & 50 & 18.4 & 15 & 11.0 & 35 & 25.7 & \\
\hline Receiving treatment & & & & & & & 0.347 \\
\hline Yes & 240 & 88.2 & 123 & 90.4 & 117 & 86.0 & \\
\hline No & 32 & 11.8 & 13 & 9.6 & 19 & 14.0 & \\
\hline Treatment duration (years) & & & & & & & 0.228 \\
\hline$<1$ & 49 & 18.0 & 21 & 15.4 & 28 & 20.6 & \\
\hline $1-5$ & 92 & 33.8 & 49 & 36.0 & 43 & 31.6 & \\
\hline$>5$ & 92 & 33.8 & 55 & 40.4 & 46 & 33.8 & \\
\hline Not applicable & 30 & 11.0 & 11 & 8.1 & 19 & 14.0 & \\
\hline PHQ-9: Depression Scale & & & & & & & $<0.001$ \\
\hline None/low & 120 & 44.1 & 80 & 58.8 & 40 & 29.4 & \\
\hline Moderate & 120 & 44.1 & 41 & 30.1 & 79 & 58.1 & \\
\hline High & 32 & 11.8 & 15 & 11.0 & 17 & 12.5 & \\
\hline $\begin{array}{l}\text { PHQ-15: Somatic Symptom Severity } \\
\text { Scale }\end{array}$ & & & & & & & $<0.001$ \\
\hline None/low & 66 & 24.3 & 50 & 36.8 & 16 & 11.8 & \\
\hline Moderate & 165 & 60.7 & 73 & 53.7 & 92 & 67.6 & \\
\hline High & 41 & 15.1 & 13 & 9.6 & 28 & 20.6 & \\
\hline GAD-7: Anxiety Severity Scale & & & & & & & $<0.001$ \\
\hline None/low & 94 & 34.6 & 64 & 47.1 & 30 & 22.1 & \\
\hline Moderate & 116 & 42.6 & 56 & 41.2 & 60 & 44.1 & \\
\hline High & 62 & 22.8 & 16 & 11.8 & 46 & 33.8 & \\
\hline General Self-Efficacy Scale & & & & & & & $<0.001$ \\
\hline Low & 98 & 36.0 & 30 & 22.1 & 68 & 50.0 & \\
\hline High & 174 & 64.0 & 106 & 77.9 & 68 & 50.0 & \\
\hline $\begin{array}{l}\text { Multidimensional Perceived Scale of } \\
\text { Social Support }\end{array}$ & & & & & & & 0.045 \\
\hline Low & 52 & 19.1 & 19 & 14.0 & 33 & 24.3 & \\
\hline High & 220 & 80.9 & 117 & 86.0 & 103 & 75.7 & \\
\hline
\end{tabular}

GI/GU = gastrointestinal/genitourinary.

$J \mathrm{D}=$ Jordanian dinar.

\section{Risk factors for depression among female Jordanians and Syrians}

Without adjusting for other covariates, we found that the female Syrian refugees had 2.73 (95\% CI: 1.71-4.39) times the odds of reporting higher levels of depression than the Jordanian women. However, after adjusting for age, monthly income, spouse employment status, GI/GU disorder status, and perceived self-efficacy, we found that the Syrians were not at statistically significantly higher odds of reporting higher levels of depression than the Jordanians (OR $=1.47 ; 95 \%$ CI: 0.81-2.67) (Table 3). Women with unemployed spouses had 130\% greater odds
$(\mathrm{OR}=2.30 ; 95 \% \mathrm{CI}: 1.26-4.25)$ of reporting higher levels of depression than women with employed spouses. Furthermore, women who had a GI or GU disorder had almost double the odds (OR $=1.96 ; 95 \%$ CI: 1.06-3.63) of having higher levels of depression than women without these diseases. We also identified that women with none/low self-efficacy had nearly double the odds of experiencing higher levels of depression than women with high self-efficacy (OR $=1.99 ; 95 \% \mathrm{CI}$ : 1.19-3.32). Increased chronic disease burden, chronic disease treatment status or length, and perceived social support were not predictive of increased depression levels. 


\begin{tabular}{|c|c|c|c|c|c|c|c|c|c|c|c|c|c|c|}
\hline \multirow[t]{3}{*}{ Characteristic } & \multicolumn{7}{|c|}{ Jordanian women, depression level } & \multicolumn{7}{|c|}{ Syrian women, depression level } \\
\hline & \multicolumn{2}{|c|}{ None/low } & \multicolumn{2}{|c|}{ Moderate } & \multicolumn{2}{|c|}{ High } & \multirow[t]{2}{*}{ P-value } & \multicolumn{2}{|c|}{ None/low } & \multicolumn{2}{|c|}{ Moderate } & \multicolumn{2}{|c|}{ High } & \multirow[t]{2}{*}{ P-value } \\
\hline & No. & $\%$ & No. & $\%$ & No. & $\%$ & & No. & $\%$ & No. & $\%$ & No. & $\%$ & \\
\hline Total & 80 & 58.8 & 41 & 30.1 & 15 & 11.0 & & 40 & 29.4 & 79 & 58.1 & 17 & 12.5 & \\
\hline Age (years) & & & & & & & 0.15 & & & & & & & 0.106 \\
\hline $18-34$ & 18 & 50.0 & 11 & 30.6 & 7 & 19.4 & & 12 & 38.7 & 13 & 41.9 & 6 & 19.4 & \\
\hline $35-50$ & 62 & 62.0 & 30 & 30.0 & 8 & 8.0 & & 28 & 26.7 & 66 & 62.9 & 11 & 10.5 & \\
\hline Marital status & & & & & & & 0.643 & & & & & & & 0.238 \\
\hline Not married & 19 & 52.8 & 13 & 36.1 & 4 & 11.1 & & 4 & 40.0 & 3 & 30.0 & 3 & 30.0 & \\
\hline Married & 61 & 61.0 & 28 & 28.0 & 11 & 11.0 & & 36 & 28.6 & 76 & 60.3 & 14 & 11.1 & \\
\hline No. of family members & & & & & & & 0.075 & & & & & & & 0.541 \\
\hline $1-6$ & 58 & 54.7 & 37 & 34.9 & 11 & 10.4 & & 24 & 26.4 & 55 & 60.4 & 12 & 13.2 & \\
\hline $7-12$ & 22 & 73.3 & 4 & 13.3 & 4 & 13.3 & & 16 & 35.6 & 24 & $53 \cdot 3$ & 5 & 11.1 & \\
\hline Education level & & & & & & & 0.429 & & & & & & & 0.363 \\
\hline None/primary & 2 & 33.3 & 3 & 50.0 & 1 & 16.7 & & 21 & 29.6 & 40 & 56.3 & 7 & 10.8 & \\
\hline More than primary & 78 & 60.0 & 38 & 29.2 & 14 & 10.8 & & 19 & 29.2 & 39 & 60.0 & 6 & 9.4 & \\
\hline Employed & & & & & & & 0.555 & & & & & & & 0.299 \\
\hline Yes & 21 & 53.8. & 12 & 30.8 & 6 & 15.4 & & 1 & 100.0 & 0 & 0 & 0 & 0 & \\
\hline No & 59 & 60.8 & 29 & 29.9 & 9 & 9.3 & & 39 & 28.9 & 79 & 58.5 & 17 & 12.6 & \\
\hline Income level (JD) & & & & & & & 0.014 & & & & & & & 0.634 \\
\hline$<200$ & 79 & 60.8 & 36 & 27.7 & 15 & 11.5 & & 22 & 31.9 & 40 & 58.0 & 7 & 10.1 & \\
\hline$\geq 200$ & 1 & 16.7 & 5 & 83.3 & 0 & 0.0 & & 18 & 26.9 & 39 & 58.2 & 10 & 14.9 & \\
\hline Housing status & & & & & & & 0.033 & & & & & & & NA \\
\hline Own & 51 & 63.0 & 25 & 30.9 & 5 & 6.2 & & 0 & 0.0 & 0 & 0.0 & 0 & 0.0 & \\
\hline Rent & 27 & 55.1 & 12 & 24.5 & 10 & 20.4 & & 40 & 29.4 & 79 & 58.1 & 17 & 12.5 & \\
\hline Other & 2 & 33.1 & 4 & 66.7 & 0 & 0.0 & & 0 & 0.0 & 0 & 0.0 & 0 & 0.0 & \\
\hline Spouse employed & & & & & & & 0.689 & & & & & & & 0.006 \\
\hline Yes & 56 & 62.9 & 24 & 27.0 & 9 & 10.1 & & 21 & 37.5 & 33 & 58.9 & 2 & 3.6 & \\
\hline No & 5 & 45.5 & 4 & 36.4 & 2 & 18.2 & & 13 & 19.1 & 43 & 63.2 & 12 & 17.6 & \\
\hline NA & 19 & 52.8 & 13 & 36.1 & 4 & 11.1 & & 6 & 50.0 & 3 & 25.0 & 3 & 25.0 & \\
\hline Spouse occupation & & & & & & & 0.065 & & & & & & & 0.017 \\
\hline Government & 24 & 53.3 & 13 & 28.9 & 8 & 17.8 & & 0 & 0.0 & 0 & 0.0 & 0 & 0.0 & \\
\hline Private/agricultural & 33() & 73.3 & 11 & 24.4 & 1 & 2.2 & & 21 & 38.2 & 33 & 58.9 & 2 & 3.6 & \\
\hline NA & 23 & 50.0 & 17 & 37.0 & 6 & 13.0 & & 19 & 23.8 & 46 & 57.5 & 15 & 18.8 & \\
\hline Disease burden & & & & & & & 0.80 & & & & & & & 0.363 \\
\hline 1 disease & 52 & 57.1 & 28 & 30.8 & 11 & 12.1 & & 22 & 34.4 & 36 & 56.3 & 6 & 9.4 & \\
\hline$>1$ disease & 28 & 62.2 & 13 & 28.9 & 4 & 8.9 & & 18 & 25.0 & 43 & 59.7 & 11 & $15 \cdot 3$ & \\
\hline Cardiometabolic disease & & & & & & & 0.37 & & & & & & & 0.802 \\
\hline Yes & 53 & 58.2 & 30 & 33.0 & 8 & 8.8 & & 18 & 26.9 & 40 & 59.7 & 9 & 13.4 & \\
\hline No & 27 & 60.0 & 11 & 24.4 & 7 & 15.6 & & 22 & 31.9 & 39 & 56.5 & 8 & 11.6 & \\
\hline Respiratory disease & & & & & & & 0.723 & & & & & & & 0.959 \\
\hline Yes & 71 & 57.7 & 38 & 30.9 & 14 & 11.4 & & 35 & 29.7 & 68 & 57.6 & 15 & 12.7 & \\
\hline No & 9 & 69.2 & 3 & 23.1 & 1 & 7.7 & & 5 & 27.8 & 11 & 61.1 & 2 & 11.1 & \\
\hline $\begin{array}{l}\text { Neurologic disease/ } \\
\text { cancer }\end{array}$ & & & & & & & 0.714 & & & & & & & 0.989 \\
\hline Yes & 70 & 59.3 & 36 & 30.5 & 12 & 10.2 & & 38 & 29.5 & 75 & 58.1 & 16 & 12.4 & \\
\hline No & 10 & 55.6 & 5 & 27.8 & 3 & 16.7 & & 2 & 28.6 & 4 & 57.1 & 1 & 14.3 & \\
\hline Rheumatologic disease & & & & & & & 0.407 & & & & & & & 0.547 \\
\hline Yes & 58 & 60.4 & 26 & 27.1 & 12 & 12.5 & & 24 & 33.3 & 40 & 55.6 & 8 & 11.1 & \\
\hline No & 22 & 55.0 & 15 & 37.5 & 3 & 7.5 & & 16 & 25.0 & 39 & 60.9 & 9 & 14.1 & \\
\hline
\end{tabular}


Table 2 Distribution of sociodemographic and health characteristics among 272 Jordanian and Syrian women in Amman, 2017 (concluded)

\begin{tabular}{|c|c|c|c|c|c|c|c|c|c|c|c|c|c|c|}
\hline \multirow[t]{3}{*}{ Characteristic } & \multicolumn{7}{|c|}{ Jordanian women, depression level } & \multicolumn{7}{|c|}{ Syrian women, depression level } \\
\hline & \multicolumn{2}{|c|}{ None/low } & \multicolumn{2}{|c|}{ Moderate } & \multicolumn{2}{|c|}{ High } & \multirow[t]{2}{*}{ P-value } & \multicolumn{2}{|c|}{ None/low } & \multicolumn{2}{|c|}{ Moderate } & \multicolumn{2}{|c|}{ High } & \multirow[t]{2}{*}{ P-value } \\
\hline & No. & $\%$ & No. & $\%$ & No. & $\%$ & & No. & $\%$ & No. & $\%$ & No. & $\%$ & \\
\hline Endocrine disease & & & & & & & 0.872 & & & & & & & 0.857 \\
\hline Yes & 54 & 57.4 & 29 & 30.9 & 11 & 11.7 & & 33 & 28.7 & 67 & 58.3 & 15 & 13.0 & \\
\hline No & 26 & 61.9 & 12 & 28.6 & 4 & 9.5 & & 7 & 33.3 & 12 & 57.1 & 2 & 9.5 & \\
\hline GI/GU disease & & & & & & & 0.631 & & & & & & & 0.003 \\
\hline Yes & 72 & 59.5 & 35 & 28.9 & 14 & 11.6 & & 33 & 32.7 & 61 & 60.4 & 7 & 6.9 & \\
\hline No & 8 & 53.3 & 6 & 40.0 & 1 & 6.7 & & 7 & 20.0 & 18 & 51.4 & 10 & 28.6 & \\
\hline Receiving treatment & & & & & & & 0.058 & & & & & & & 0.844 \\
\hline Yes & 74 & 60.2 & 38 & 30.9 & 11 & 8.9 & & 34 & 29.1 & 69 & 59.0 & 14 & 12.0 & \\
\hline No & 6 & 46.2 & 3 & 23.1 & 4 & 30.8 & & 6 & 31.6 & 10 & 52.6 & 3 & 15.8 & \\
\hline $\begin{array}{l}\text { Treatment duration } \\
\text { (years) }\end{array}$ & & & & & & & 0.079 & & & & & & & 0.556 \\
\hline$>1$ & 13 & 61.9 & 8 & 38.1 & 0 & 0.0 & & 5 & 17.9 & 21 & 75.0 & 2 & 7.1 & \\
\hline $1-5$ & 32 & 65.3 & 13 & 26.5 & 4 & 8.2 & & 13 & 30.2 & 23 & 53.5 & 7 & 16.3 & \\
\hline$>5$ & 31 & 56.4 & 17 & 30.9 & 7 & 12.7 & & 16 & 34.8 & 25 & 54.3 & 5 & 10.9 & \\
\hline NA & 4 & 36.4 & 3 & 27.3 & 4 & 36.4 & & 6 & 31.6 & 10 & 52.6 & 3 & 15.8 & \\
\hline PHQ-15 severity & & & & & & & $<0.001$ & & & & & & & $<0.001$ \\
\hline Low & 35 & 70.0 & 14 & 28.0 & 1 & 2.0 & & 10 & 62.5 & 6 & 37.5 & 0 & 0.0 & \\
\hline Moderate & 43 & 58.9 & 22 & 30.1 & 8 & 11.0 & & 27 & 29.3 & 57 & 62.0 & 8 & 8.7 & \\
\hline High & 2 & 15.4 & 14 & 28.0 & 6 & 46.2 & & 3 & 10.7 & 16 & 57.1 & 9 & 32.1 & \\
\hline GAD-7 severity & & & & & & & $<0.001$ & & & & & & & $<0.001$ \\
\hline None/low & 57 & 89.1 & 7 & 10.9 & 0 & 0.0 & & 21 & 70.0 & 9 & 30.0 & 0 & 0.0 & \\
\hline Moderate & 23 & 41.1 & 29 & 51.8 & 4 & 7.1 & & 14 & 23.3 & 45 & 75.0 & 1 & 1.7 & \\
\hline High & 0 & 0.0 & 5 & 31.3 & 11 & 68.8 & & 5 & 10.9 & 25 & 54.3 & 16 & 34.8 & \\
\hline GSE scale score & & & & & & & 0.041 & & & & & & & 0.183 \\
\hline None/ low & 68 & 64.2 & 29 & 27.4 & 9 & 8.5 & & 22 & 32.4 & 41 & 60.3 & 5 & 7.4 & \\
\hline High & 12 & 40.0 & 12 & 40.0 & 6 & 20.0 & & 18 & 26.5 & 38 & 55.9 & 12 & 17.6 & \\
\hline MSPSS score & & & & & & & 0.008 & & & & & & & $<0.001$ \\
\hline None/low & 8 & 42.1 & 5 & 26.3 & 6 & 31.6 & & 7 & 21.2 & 14 & 42.4 & 12 & 36.4 & \\
\hline High & 72 & 61.5 & 36 & 30.8 & 9 & 7.7 & & 33 & 32.0 & 65 & 63.1 & 5 & 4.9 & \\
\hline
\end{tabular}

\section{JD = Jordanian dinars.}

NA = not applicable.

GI/GU = gastrointestinal/genitourinary

PHQ-15 = Patient Health Questionnaire-15.

GAD-7 = Generalized Anxiety Disorder-7.

GSE $=$ General Self-Efficacy.

MSPSS = Multidimensional Scale of Perceived Social Support.

\section{Discussion}

This study compared the prevalence and risk factors of depression among female Syrian refugees and Jordanians with chronic disease. We found that approximately half of the total study population reported moderate to high depression, which was comorbid in less than half of the Jordanians and nearly three-quarters of the Syrian refugees. This prevalence of depression is substantially higher than reported in prior studies, which have found depression in $35 \%$ of Syrian refugees suffering from chronic diseases (7), with depression prevalence among all refugees varying from $3 \%$ to $85.5 \%(22,23)$.
Although most research examining the psychosocial needs of displaced Syrians in Jordan is carried out in refugee camps, more than $80 \%$ of registered Syrian refugees live in non-camp settings, demonstrating a need to understand the burden of depression among non-camp dwelling refugees (1). The higher prevalence of depressive symptoms among non-camp refugees may be partially attributable to post-resettlement hardships such as finding employment restricted work permits and adapting to a new cultural, economic and political environment without the embedded social support group that a camp would afford. A study examining the connection between self-care activities and depression 


\begin{tabular}{|c|c|c|}
\hline Characteristic & $\begin{array}{l}\text { Adjusted OR } \\
(95 \% \mathrm{CI})\end{array}$ & P-value \\
\hline \multicolumn{3}{|l|}{ Age } \\
\hline $18-34$ & 1 & \\
\hline $34-50$ & $0.69(0.39-1.23)$ & 0.209 \\
\hline \multicolumn{3}{|l|}{ Monthly income (JD) } \\
\hline$\geq 200$ & 1 & \\
\hline$<200$ & $1.33(0.73-2.42)$ & 0.353 \\
\hline \multicolumn{3}{|l|}{ Employed spouse } \\
\hline Yes & 1 & \\
\hline No & $2.30(1.26-4.25)$ & $0.007^{\mathrm{a}}$ \\
\hline Not applicable (not married) & $0.892(0.71-1.13)$ & 0.331 \\
\hline \multicolumn{3}{|l|}{ Nationality } \\
\hline Jordanian & 1 & \\
\hline Syrian & $1.47(0.81-2.67)$ & 0.203 \\
\hline \multicolumn{3}{|l|}{ GI/GU disease diagnosis } \\
\hline No & 1 & \\
\hline Yes & $1.96(1.06-3.63)$ & $0.031^{\mathrm{a}}$ \\
\hline \multicolumn{3}{|l|}{ Perceived self-efficacy } \\
\hline High & 1 & \\
\hline Low & $1.99(1.19-3.32)$ & $0.008^{a}$ \\
\hline
\end{tabular}

$\mathrm{OR}=$ odds ratio; $\mathrm{CI}=$ confidence interval; $J \mathrm{D}=$ Jordanian dinars; $\mathrm{GI} / \mathrm{GU}=$ gastrointestinal $/$ genitourinary.

aVariables statistically significantly associated with level of depression, $P \leq 0.05$.

among Jordanians with diabetes reported that $70 \%$ of participants had depressive symptoms (6). There has been limited research on the link between mental health and chronic disease among Jordanians.

Our sample size calculation suggests that our study is adequately powered; however, sampling at clinics and hospitals serving primarily low-income Jordanians and Syrians may have resulted in disproportionately high levels of depression compared with the overall population. Moreover, an all-female cohort may have increased depression prevalence $(3,4)$. In addition to potential hormonal contributors, women are predisposed to factors that increase the likelihood of depression (4). Among Middle Eastern women, these may include financial dependence, sexual violence, changing roles of women in Arab culture, orthodox customs and an increased willingness to report depressive symptoms to health care providers (4). Furthermore, depression is more common among displaced women than men (24).

A number of factors were predictive of higher levels of depression among both cohorts. For instance, spouse unemployment was highly predictive of increased depression levels. Previous research suggests that difficulty managing chronic conditions or caring for family members with mental and physical illnesses contributed to unemployment among Iraqi refugees (25). The relationship between unemployment and financial distress, leading to continued unemployment, is also well documented (26). However, few studies have reported the relationship between mental health of the patient and his or her spouse's employment status. Interestingly, income was not a significant predictor of depression in our population.

We found that a GI/GU disease diagnosis was associated with an increased likelihood of higher depression levels. Depression is a known contributor to the pathogenesis of various gastrointestinal illnesses including irritable bowel syndrome, inflammatory bowel disease and ulcerative colitis, and urologic diagnoses such as incontinence $(27,28)$. Chronic inflammation, identified through biomarkers interlukin-6 and C-reactive protein, may play a role in the relationship between depression and inflammatory diseases (29). In addition, a study demonstrated that patients with multiple lower urinary tract symptoms experienced high levels of psychiatric morbidity (30).

Low perceived self-efficacy was associated with higher levels of depression. A strong sense of self-efficacy, especially helpful among vulnerable populations exposed to frequent adversity, has been linked to improved health and social integration (31). Highly efficacious patients are more likely to perceive obstacles as challenges to be mastered, in turn lowering stress and reducing susceptibility to depression (32). A recent study found an association between a higher perceived ability to handle traumatic events and improved psychological adaptation among Syrian refugees (33). We did not find any statistically significant association between low perceived social support, an established contributor to mental health issues (34), and higher levels of depression. This suggests that among female Syrians and Jordanians with chronic disease perceived self-determination may play a greater role in reducing depression than the extent of one's social network. For instance, showing refugees traumatic pictures after recalling self-efficacious memories has been shown to decrease distress (35). Increasing accessibility of factors that may reduce postmigration stressors, such as loss of community support, lack of employment opportunities, social isolation, and cultural differences, may improve self-efficacy and coping with adversity (36).

While Syrians have greater odds of experiencing higher levels of depression than Jordanians, we found that after adjusting for sociodemographic characteristics, disease status and levels of self-efficacy, there was no longer a significant difference between these 2 populations. Thus, by changing some of the modifiable risk factors of depression, as identified in this study, it may be possible to make meaningful reductions in the odds of experiencing depression in this population. Specifically, investment in public health interventions that are aimed at improving self-efficacy in the Jordanian and Syrian populations will likely reduce rates of depression. Examples of such interventions include positive selfefficacy induction, in which refugees retrieve masteryrelated autobiographical memories shown to promote 
greater distress tolerance (35), and providing role models for newly arrived refugees, suggested in a self-efficacy study among Afghan and Kurdish refugees (32). Labour laws limiting the provision of work permits for Syrian refugees in Jordan may be influencing the likelihood of higher levels of depression in Syrians (37), as indicated by the increased odds of higher levels of depression among women whose husbands were unemployed and the finding that there was a greater proportion of unemployed husbands among Syrian than Jordanian women in our sample. Lastly, providing women's health services to vulnerable populations, as well as mental health services for survivors of sexual traumatic events, remains critical. Potential interventions for Jordanians include mindfulness and increased social support, which have been shown to improve psychological well-being and quality of life among Jordanian women with breast cancer (38).

This study had several limitations. We employed self-reporting instruments, leading to possible recall and self-report bias. In addition, the cross-sectional nature of our study precludes causal explanations related to risk factors. Predictors were selected based on prior studies, but others may have been selected, including past trauma, personal loss and lack of access to food or water $(23,36)$. Finally, we used convenience sampling, which brings all the limitations inherent in a non-probability sample. However, these limitations are mitigated by the pilot study design, which is intended to assist with future studies employing probability sampling, and is not intended to be representative of the broader population.

\section{Conclusion}

Comparing factors influencing depression in female Syrian refugees and their Jordanian counterparts allows mental health interventions and chronic disease management to be tailored to differences, and similarities, among local and displaced communities. Spouse unemployment, lower perceived self-efficacy, and diagnosis of GI/GU disease were associated with increased rates of depression regardless of nationality. These groups should be targeted for depression prevention and treatment. Such findings are important considerations for mental health programs and for developing comprehensive interventions designed to address individual patient needs.

Funding: This study was funded by the Yale School of Medicine Office of Student Research and the Wilbur Downs International Health Student Travel Fellowship.

Competing interests: None declared.

\section{Prévalence et facteurs de risque de la dépression chez les réfugiées syriennes et les Jordaniennes atteintes de maladies chroniques : une étude pilote \\ Résumé}

Contexte : Les maladies chroniques et la dépression sont les principales causes de morbidité parmi les populations déplacées et locales.

Objectifs : La présente étude vise à évaluer la prévalence et les facteurs de risque de comorbidité dépressive chez les femmes jordaniennes et syriennes atteintes de maladies chroniques connues.

Méthodes : Afin de fournir des données probantes pour la planification des interventions de santé mentale, nous avons mené une enquête transversale sur les déterminants de la dépression chez les réfugiées syriennes et les Jordaniennes atteintes de maladies chroniques vivant à Amman. De juin à août 2017, 272 réfugiées syriennes et Jordaniennes atteintes de maladies chroniques ont été recrutées dans quatre dispensaires d'Amman. Nous avons comparé les caractéristiques démographiques et sanitaires ainsi que le niveau de dépression et identifié des prédicteurs de dépression par le biais d'une régression ordinale multivariable.

Résultats : Des niveaux modérés à élevés de dépression ont été signalés chez 55,9\% des participantes, la prévalence étant de $41,1 \%$ chez les Jordaniennes et de 70,6 \% chez les Syriennes. Les Syriennes atteintes de maladies chroniques avaient 2,73 fois plus de risques de souffrir de niveaux plus élevés de dépression que leurs homologues jordaniennes. Après ajustement en fonction de l'âge, du revenu, de la situation professionnelle du conjoint, de la présence de troubles gastro-intestinaux ou génito-urinaires et de l'auto-efficacité perçue, les Syriennes n'étaient pas significativement plus susceptibles de signaler des niveaux de dépression plus élevés. Les facteurs de risque pour des niveaux de dépression plus élevés comprenaient le fait d'avoir un conjoint sans emploi, le diagnostic de troubles gastro-intestinaux ou génito-urinaires et une faible auto-efficacité perçue.

Conclusion : La comorbidité dépressive est souvent fréquente en présence de maladies physiques chroniques et a un impact délétère sur l'état de santé. Des interventions dans le domaine de la santé mentale et une prise en charge des maladies chroniques adaptées aux différences entre les communautés locales et déplacées peuvent réduire la charge de morbidité et les incapacités. 


$$
\begin{aligned}
& \text { معدل انتشار الاكتئاب في صفوف النساء الأردنيات واللاجئات السوريات المصابات بأمر اض مزمنة وعو امل الخطر } \\
& \text { المرتبطة به: در اسة تجريبية } \\
& \text { ماديسون شارب، أليسا باربيا، مأمون أهرام، رفقي محمود، كافيه خوشنود } \\
& \text { الخلفية: تُعد الأمر اض المز منة والاكتئاب من الأسباب الرئيسية التي تؤدي إلى المراضة في صفوف النازحين و السكان المحليين. }
\end{aligned}
$$

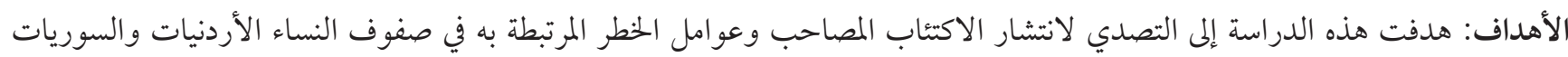

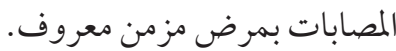

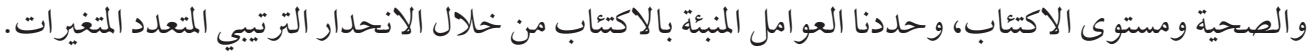

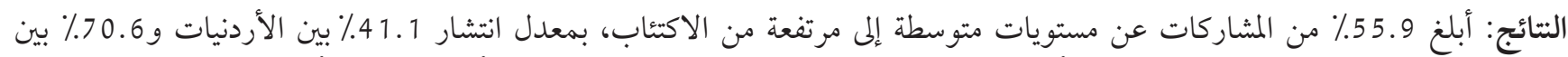

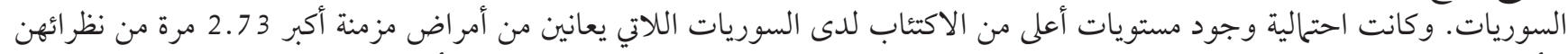

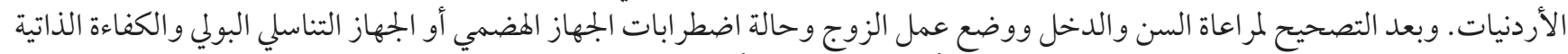

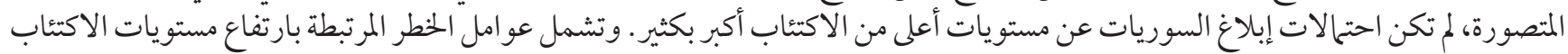

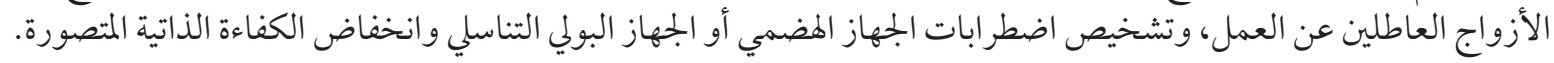

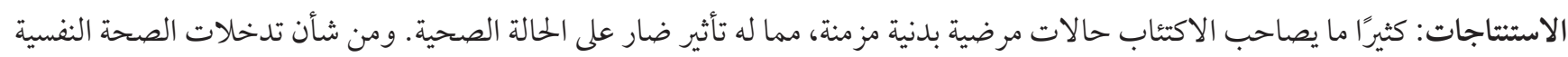

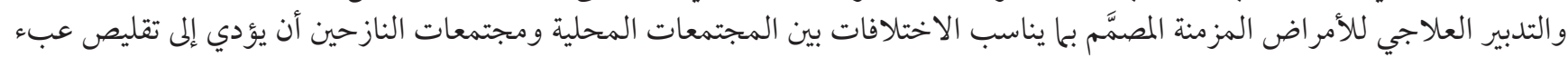
المرض والعجز.

\section{References}

1. Operational portal: refugee situation. Syria regional refugee response. Geneva: United Nations High Commissioner for Refugees; 2019 (https://data2.unhcr.org/en/situations/syria, accessed 1 January 2019).

2. Yun K, Hebrank K, Graber LK, Sullivan M-C, Chen I, Gupta J. High prevalence of chronic non-communicable conditions among adult refugees: implications for practice and policy. J Community Health. 2012;37(5):1110-8. https://doi.org/10.1007/s10900-0129552-1

3. Hollander A-C, Bruce D, Burström B, Ekblad S. Gender-related mental health differences between refugees and non-refugee immigrants--a cross-sectional register-based study. BMC Public Health. 2011;11:180. https://doi.org/10.1186/1471-2458-11-180

4. Eloul L, Ambusaidi A, Al-Adawi S. Silent epidemic of depression in women in the Middle East and North Africa region: emerging tribulation or fallacy? Sultan Qaboos University Med J. 2009;9(1):5-15. http://www.ncbi.nlm.nih.gov/pubmed/21509269

5. Al-Nsour M, Zindah M, Belbeisi A, Hadaddin R, Brown D, Walke H. Prevalence of selected chronic, noncommunicable disease risk factors in Jordan: results of the 2007 Jordan Behavioral Risk Factor Surveillance Survey. Prev Chronic Dis. 2011;9(25). https:// www.ncbi.nlm.nih.gov/pmc/articles/PMC3277383/

6. Al-Amer RM, Sobeh MM, Zayed AA, Al-Domi HA. Depression among adults with diabetes in Jordan: risk factors and relationship to blood sugar control. J Diabetes Its Complications. 2011;25(4):247-52. doi:10.1016/J.JDIACOMP.2011.03.001

7. Gammouh OS, Al-Smadi AM, Tawalbeh LI, Khoury LS. Chronic diseases, lack of medications, and depression among Syrian refugees in Jordan, 2013-2014. Prev Chronic Dis. 2015;12:140424. doi:10.5888/pcd12.140424

8. Katon W, Von Korff M, Lin E, Simon G, Ludman E, Bush T, et al. Improving primary care treatment of depression among patients with diabetes mellitus: the design of the pathways study. Gen Hosp Psychiatry. 2003;25(3):158-68. https://doi.org/10.1016/ S0163-8343(03)00013-6

9. World Bank country and lending overview. Washington DC: World Bank; 2019 (https://datahelpdesk.worldbank.org/knowledgebase/articles/906519-world-bank-country-and-lending-groups, accessed 13 March 2019).

10. About us: UNDP Jordan. Amman: United Nations Development Programme; 2019 (http://www.jo.undp.org/content/jordan/en/ home/about-us.html, accessed 10 January 2019).

11. Jordan overview. Washington DC: World Bank; 2019 (http://www.worldbank.org/en/country/jordan/overview\#1, accessed 10 April 2019).

12. Jordan overview. Washington DC: World Bank; 2019 (http://www.worldbank.org/en/country/jordan/overview\#1, accessed 10 April 2019). 
13. Nazer LH, Tuffaha H. Health care and pharmacy practice in Jordan. Canadian J Hosp Pharm. 2017;70(2):150-5. http://www.ncbi. nlm.nih.gov/pubmed/28487583

14. Health access and utilization survey: access to health services in Jordan among Syrian refugees. Geneva: United Nations High Commissioner for Refugees; 2017 (https://reliefweb.int/sites/reliefweb.int/files/resources/UNHCR-HealthAccess\%26UtilizationSurveyinJordan2017-Syrians.pdf, accessed 11 July 2020).

15. Kroenke K, Spitzer RL, Williams JB. The PHQ-9: validity of a brief depression severity measure. J Gen Intern Med. 2001;16(9):606-13. https://doi.org/10.1046/J.1525-1497.2001.016009606.X

16. Manea L, Gilbody S, McMillan D. Optimal cut-off score for diagnosing depression with the Patient Health Questionnaire (PHQ9): a meta-analysis. CMAJ. 2012;184(3):E191-6. doi:10.1503/cmaj.110829

17. Aroian K, Templin TN, Ramaswamy V. Adaptation and psychometric evaluation of the multidimensional scale of perceived social support for Arab immigrant women. Health Care Women Int. 2010;31(2):153-69. doi:10.1080/07399330903052145

18. Crandall A, Rahim HFA, Yount KM. Validation of the general self-efficacy scale among Qatari young women. East Mediterer Health J. 2015;21(12):891-6. doi:10.26719/2015.21.12.891

19. AlHadi AN, AlAteeq DA, Al-Sharif E, Bawazeer HM, Alanazi H, AlShomrani AT, et al. An Arabic translation, reliability, and validation of Patient Health Questionnaire in a Saudi sample. Ann Gen Psychiatry. 2017;16:32. doi:10.1186/s12991-017-0155-1

20. Spitzer RL, Kroenke K, Williams JBW, Löwe B. A brief measure for assessing generalized anxiety disorder. Arch Int Med. 2006;166(10):1092. doi:10.1001/archinte.166.10.1092

21. Poole DN, Hedt-Gauthier B, Liao S, Raymond NA, Bärnighausen T. Major depressive disorder prevalence and risk factors among Syrian asylum seekers in Greece. BMC Public Health. 2018;18(1):908. doi:10.1186/s12889-018-5822-x

22. Feyera F, Mihretie G, Bedaso A, Gedle D, Kumera G. Prevalence of depression and associated factors among Somali refugee at Melkadida camp, southeast Ethiopia: a cross-sectional study. BMC Psychiatry. 2015;15(1):171. doi:10.1186/s12888-015-0539-1

23. Steel Z, Chey T, Silove D, Marnane C, Bryant RA, van Ommeren M. Association of torture and other potentially traumatic events with mental health outcomes among populations exposed to mass conflict and displacement. JAMA. 2009;302(5):537. doi:10.1001/ jama.2009.1132

24. Sheikh TL, Abdulaziz M, Agunbiade S, Joseph I, Ebiti B, Adekeye O. Correlates of depression among internally displaced persons after post-election violence in Kaduna, North Western Nigeria. J Affective Disorders. 2015;170:46-51. doi:10.1016/J. JAD.2014.08.050

25. Wright AM, Dhalimi A, Lumley MA, Jamil H, Pole N, Arnetz JE, et al. Unemployment in Iraqi refugees: the interaction of pre- and post-displacement trauma. Scand J Psychol. 2016 Dec;57(6):564-70. doi:10.1111/sjop.12320

26. Paul K. I, Moser K. Unemployment impairs mental health: meta-analyses. J Vocational Behav. 2009;74(3):264-82. doi:10.1016/J. JVB.2009.01.001

27. Moussavi S, Chatterji S, Verdes E, Tandon A, Patel V, Ustun B. Depression, chronic diseases, and decrements in health: results from the World Health Surveys. Lancet. 2007;370:851-8. doi:10.1016/So140-6736(07)61415-9

28. Zorn BH, Montgomery H, Pieper K, Gray M, Steers WD. Urinary incontinence and depression. J Urol. 1999;162:82-4. doi:10.1097/00005392-199907000-00020

29. Miller AH, Maletic V, Raison CL. Inflammation and its discontents: the role of cytokines in the pathophysiology of major depression. Biol Psychiatry. 2009;65:732-41. doi:10.1016/j.biopsych.2008.11.029

30. Coyne KS, Wein AJ, Tubaro A, Sexton CC, Thompson CL, Kopp ZS, et al. The burden of lower urinary tract symptoms: evaluating the effect of LUTS on health-related quality of life, anxiety and depression: EpiLUTS. BJU Int. 2009;103:4-11. doi:10.1111/j.1464410X.2009.08371.X

31. Scholz U, Gutiérrez Doña B, Sud S, Schwarzer R. Is general self-efficacy a universal construct. Eur J Psycholog Assess. 2002;18(3):242-51. doi:10.1027/1015-5759.18.3.242

32. Sulaiman-Hill CMR, Thompson SC. Learning to fit in: an exploratory study of general perceived self efficacy in selected refugee groups. J Immigr Minor Health. 2013;15(1):125-31. doi:10.1007/s10903-011-9547-5

33. Von Haumeder A, Ghafoori B, Retailleau J. Psychological adaptation and posttraumatic stress disorder among Syrian refugees in Germany: a mixed-methods study investigating environmental factors. Eur J Psychotraumatol, 2019;10(1):1686801. doi:10.1080/20 008198.2019.1686801

34. Grav S, Hellzèn O, Romild U, Stordal E. Association between social support and depression in the general population: the HUNT study, a cross-sectional survey. J Clinic Nurs. 2012;21(1-2):111-20. doi:10.1111/j.1365-2702.2011.03868.x

35. Morina N, Bryant RA, Doolan EL, Martin-Sölch C, Plichta MM, Pfaltz, et al. The impact of enhancing perceived self-efficacy in torture survivors. Depress Anxiety. 2018;35(1):58-64. doi:10.1002/da.22684

36. Seccombe K. "Beating the odds" versus "changing the odds": poverty, resilience, and family policy. J Marriage Fam. 2002;64(2):384-94. doi:10.1111/j.1741-3737.2002.00384.X

37. Sarrado O, Dunmore C. New deal on work permits helps Syrian refugees in Jordan. Geneva: United Nations High Commissioner for Refugees; 2017 (https://www.unhcr.org/news/latest/2017/10/59df254b4/new-deal-work-permits-helps-syrian-refugees-jordan. html, accessed 10 January 2019).

38. Al-Ghabeesh S, Al-Kalaldah M, Rayan A, Al-Rifai A, Al-Halaiqa F. Psychological distress and quality of life among Jordanian women diagnosed with breast cancer: the role of trait mindfulness. Eur J Cancer Care. 2019;28(5):e13082. doi:10.1111/ecc.13082 